\title{
The Ability of Self-Rated Health to Predict Mortality among Community-Dwelling Elderly Individuals Differs according to the Specific Cause of Death: Data from the NEDICES Cohort
}

\author{
Mario Fernández-Ruiz ${ }^{a}$ Juan M. Guerra-Vales ${ }^{a}$ Rocío Trincado $^{b, c}$ \\ Rebeca Fernández $^{d}$ María José Medrano ${ }^{e}$ Alberto Villarejo ${ }^{\text {b, c }}$ \\ Julián Benito-León ${ }^{b, c}$ Félix Bermejo-Parejab, ${ }^{\text {c }}$ \\ Departments of anternal Medicine and ' Neurology, Hospital Universitario '12 de Octubre', Instituto de Investigación \\ Hospital '12 de Octubre' (i+12), Universidad Complutense, 'Centro de Investigación Biomédica en Red sobre \\ Enfermedades Neurodegenerativas (CIBERNED), Instituto de Salud Carlos III, dUnit of Neurology, Clínica 'La Luz', and \\ eNational Centre for Epidemiology, Instituto de Salud Carlos III, Madrid, Spain
}

\section{Key Words}

Self-rated health - Causes of death · Elderly · Mortality · NEDICES Study

\begin{abstract}
Background: The biomedical and psychosocial mechanisms underlying the relationship between self-rated health (SRH) and mortality in elderly individuals remain unclear. Objective: To assess the association between different measurements of subjective health (global, age-comparative, and time-comparative SRH) and cause-specific mortality. Methods: Neurological Disorders in Central Spain (NEDICES) is a prospective population-based survey of the prevalence and incidence of major age-associated conditions. Data on demographic and health-related variables were collected from 5,278 subjects ( $\geq 65$ years) in the baseline questionnaire. Thirteen-year mortality and cause of death were obtained from the National Death Registry. Adjusted hazard ratios (aHR) for SRH and all-cause and cause-specific mortality
\end{abstract}

were estimated by Cox proportional hazard models. Results: At baseline, 4,958 participants (93.9\%) answered the SRH questionnaire. At the end of follow-up, 2,468 (49.8\%) participants had died, of whom 723 (29.2\%) died from cardiovascular diseases, 609 (24.7\%) from cancer, and 359 (14.5\%) from respiratory diseases. Global SRH independently predicted all-cause mortality (aHR for 'poor or very poor' vs. 'very good' category: 1.39; 95\% confidence interval (Cl): 1.15-1.69). Analysis of cause-specific mortality revealed that global SRH was an independent predictor for death due to respiratory diseases (aHR for 'poor or very poor' vs. 'very good' category: 2.61 ; 95\% Cl: 1.55-4.39), whereas age-comparative SRH exhibited a gradient effect on the risk of death due to stroke. Time-comparative SRH provided small additional predictive value. Conclusions: The predictive ability of SRH for mortality largely differs according to the specific cause of death, with the strongest associations found for respiratory disease and stroke mortality.

Copyright $\odot 2013$ S. Karger AG, Basel

\section{KARGER}

E-Mail karger@karger.com

www.karger.com/ger
(C) 2013 S. Karger AG, Basel

0304-324X/13/0594-0368\$38.00/0
Mario Fernández-Ruiz, MD

Department of Internal Medicine, Hospital Universitario '12 de Octubre'

Centro de Actividades Ambulatorias, planta 2a ${ }^{\text {a }}$, bloque D

Avda. de Córdoba, s/n, CP 28041, ES-28041 Madrid (Spain)

E-Mail mario_fdezruiz@yahoo.es 


\section{Introduction}

Since the preliminary evidence reported three decades ago by Mossey and Shapiro [1], a large number of both community- and clinical-based studies have demonstrated a strong and persistent association between self-rated health (SRH) and mortality [2-6]. A meta-analysis including data from 22 studies concluded that persons with 'poor' SRH had a 2-fold higher mortality risk compared to persons with 'excellent' SRH. This association remained robust even after adjusting for covariates such as functional status, depression, and objective burden of comorbidity, and irrespective of gender, length of followup, or country of origin [7]. The subjective assessment of health status has also been shown to accurately predict mortality among patients with specific conditions $[8,9]$, as well as other health-related outcomes such as future changes in functional ability [10] or utilization of healthcare resources [11].

Despite this cumulative body of knowledge, the biomedical and psychosocial mechanisms underlying the relationship between SRH and mortality remain largely unclear [12]. It has been reported that its strength may be tempered by a number of demographic $[13,14]$ and socioeconomic variables $[5,15]$, either suggesting the influence of confounding factors or actually reflecting variations in the ability of SRH to predict subsequent mortality as a whole. An intuitive insight into this question should come from breaking down the overall effect of SRH on specific causes of death rather than just analyzing all-cause mortality. The association between SRH and death is more plausible in chronic conditions with a protracted course and greater impact on daily life (e.g., chronic obstructive pulmonary disease, COPD) than in more aggressive types of disease with a worse prognosis in the mid-term (e.g., cancer) or a mainly silent course (e.g., myocardial infarction). In addition, the strength of this association might be weaker in patients with poor self-awareness of own diagnoses (e.g., dementia). On the other hand, a bidirectional influence may be hypothesized in which low perceived health status would have affective and behavioral effects (lack of physical exercise, noncompliance with treatment) with a differential impact depending on the natural history of the underlying condition (e.g., greater in circulatory or respiratory than in malignant diseases) $[8,9]$. To confirm such hypotheses the analysis of specific causes of death should offer a valuable proxy for the main condition from among the number of comorbidities accumulated throughout the subject's life. However, and surprisingly in view of the exten-

Self-Rated Health and Cause-Specific Mortality sive research performed in this field, only a few papers have taken into account cause-specific mortality $[4,16-$ 22].

Another potential source of variability in the literature lies in the different responders' expectations when they instinctively compare themselves with others of the same age, or when they keep in mind how they were becoming ill [12]. It has been theorized that the process of rating one's own health status contains an implicit social comparison with those whose conditions are perceived as even worse [19]. Furthermore, the very nature of the chronic condition that ultimately leads to death could modulate the subject's own perception of improvement or worsening over time and, therefore, the magnitude of the relationship between SRH and the risk of death. Unfortunately there is a scarcity of research on how these different evaluations of SRH (global, age-comparative, and time-comparative) affect its predictive value on mortality.

Thus, the present study was aimed to investigate the role of SRH - as assessed by one absolute and two comparative questions - in predicting mortality due to a number of specific causes in a broad population-based cohort of individuals aged 65 years and older living in three communities in central Spain.

\section{Methods}

\section{Study Setting and Population}

The present study constitutes a subanalysis of data derived from the Neurological Disorders in Central Spain (NEDICES) Study, a longitudinal, population-based survey of the prevalence, incidence and determinants of major age-associated neurological conditions in persons aged 65 years and older. The NEDICES Study went into one well-defined area of Spain (central Spain) and sampled three different communities: Las Margaritas, a workingclass neighborhood in Getafe (Greater Madrid); Lista, a professional-class neighborhood in Salamanca district (central Madrid); and Arévalo county (Ávila), an agricultural zone $125 \mathrm{~km}$ northwest of Madrid. The registered study population consisted of 6,395 individuals, but 481 were ineligible (census issues, incorrect address, or death), leaving 5,914 eligible subjects, of whom 5,278 (89.2\%) were evaluated at baseline. All procedures were approved by the Ethical Standards Committees on Human Experimentation at the University Hospitals ' 12 de Octubre' (Madrid) and 'La Princesa' (Madrid). Written (signed) informed consent was obtained from all participants.

\section{Study Design and Measurements}

The methodology of the NEDICES Study has been described elsewhere [23-25]. In brief, face-to-face evaluations were performed at baseline (1994-1995) and then at follow-up (19971998). The baseline survey was performed with a detailed ques- 
tionnaire administered to enrollees by social workers, student nurses, and general physicians. This interview comprised a 500item structured questionnaire assessing demographic information, variables about lifestyle (physical activity, smoking habits, and consumption of alcohol), all current medications (those used during the previous week), and objective health status. The latter was evaluated through the question 'Has a medical provider ever told you that you have any of the following?', which was followed by a list of 13 diseases or chronic conditions. Three response choices were offered for each question: 'yes', 'no', or 'I don't know' (regarded as negative responses for the purposes of the present study). SRH was evaluated by means of 3 different questions, similar to those formulated in the National Spanish Health Survey [26]. The first question was: 'In general terms, how would you describe your health: very good, good, fair, poor, or very poor?' (global SRH). The second one was: 'How would you describe your health compared to others of your age and gender: much better, better, similar, worse, or much worse?' (age-comparative SRH). Due to low relative frequencies, the 2 lowest categories in each question were merged into one for the survival analyses. Finally, the third one was: 'How would you describe your health as compared to one year ago: much better, better, similar, worse, or much worse?' (timecomparative SRH). In this case the extreme categories were combined to form a 3-level variable. If not otherwise mentioned, analyses refer to global SRH. Education level was categorized in 4 levels ('no schooling and illiterate', 'can write and read', 'primary studies', or 'secondary studies and higher'). The screening instruments for dementia included a cognitive test (a 37-item version of the Mini-Mental State Examination, MMSE) [27] and the Pfeffer Functional Activities Questionnaire (FAQ) [28]. The presence of depressive symptoms was assessed by self-report, using a single screening question ('Do you suffer from depression?'). Such approach has similarly been utilized in other previous studies of depression [29]. Participants who screened positive for any neurological disease (e.g., Parkinson's disease, essential tremor, dementia or stroke) were further examined by one senior neurologist to obtain a definitive diagnosis $[23,24]$. No patient was excluded on the basis of the presence of cognitive impairment. In such a case, the questionnaire was completed with the support of the participant's main carer and general practitioner. A short form of this study questionnaire was mailed to subjects who refused or were unavailable for face-to-face screening. During the second evaluation (1997-1998), the same methods were used and global SRH was also assessed by means of the same question. Nevertheless, for the present study we will only use data on SRH obtained at the baseline evaluation (1994-1995).

\section{Ascertainment of Mortality}

The mortality of the NEDICES Study cohort was evaluated by means of the National Death Registry of the Spanish National Statistics Institute (Instituto Nacional de Estadística), with the appropriate permission. In Spain, all deceased individuals receive a death certificate, completed by a doctor, at the time of death. The certificate is then sent to the court local police authority in the municipality where the person had been living, and the information is collected in the National Registry. The cause of death was classified into 8 main categories according to the 9th revision of the International Classification of Diseases (available at http:// www.cdc.gov/nchs/icd/icd9.htm): 'myocardial infarction', 'other cardiovascular diseases' (pulmonary embolism, congestive heart failure, heart or aortic rupture, and asystole), 'stroke', 'dementia', 'other neurological disorders', 'cancer', 'respiratory diseases', and 'other causes' (e.g., infections, trauma, genitourinary, or gastrointestinal disorders). The survival interval for the mortality analysis of each participant was analyzed from the day in which the baseline interview was performed (1994-1995) until the day of death, or until December 31, 2007 for living subjects (about 13-year followup).

\section{Statistical Analysis}

All statistical analyses were performed using SPSS version 15.0 (Statistical Package for the Social Sciences Inc ${ }^{\circledR}$, Chicago, Ill., USA). Qualitative variables were expressed as absolute and relative frequencies. Quantitative data were shown as mean \pm standard deviation (SD), or median (25th-75th percentile or range). Categorical variables were compared using the $\chi^{2}$ test, whereas the one-way ANOVA test (or Kruskal-Wallis test when the assumption of normality did not hold) was applied for continuous variables. The associations between different categories of SRH at baseline and both all-cause and cause-specific 13-year mortality were assessed using Cox proportional hazards models. Various Cox models with increasing number of covariates were carried out to evaluate the residual effect on mortality of different SRH-related questions. From a starting model (1) adjusted for age and gender, we sequentially built additional models that included (2) sociodemographic variables, health-affecting habits and functional status, and (3) number of chronic comorbidities. Adjusted hazard ratios (aHRs) were shown with the $95 \%$ confidence intervals $(95 \% \mathrm{CI})$. All p values were two-tailed, and we considered $\mathrm{p}<0.05$ as significant.

\section{Results}

Overall, the study cohort comprised a total of 5,278 participants, of which 320 (6.1\%) with missing global SRH data were excluded. As compared to the remaining participants, these subjects were older $(77.9 \pm 8.1$ vs. 74.1 \pm 6.8 years; $\mathrm{p}<0.001)$, had higher functional dependence (median (25th-75th percentile) values in the Pfeffer FAQ scale: $16.9(0.2-33)$ vs. $0(0-3)$; $\mathrm{p}<0.001)$ and higher prevalence of dementia (18.1 vs. $5.0 \%$; $p<0.001)$, and were more frequently illiterate $(23.5$ vs. $13.0 \%$; $<<0.001)$.

Therefore, the final study sample comprised 4,958 subjects (2,105 men and 2,853 women), only 55 of whom (1.1\%) were institutionalized. Their demographic and clinical characteristics are shown in table 1 . At the baseline evaluation, 507 (10.2\%) individuals evaluated their global SRH as 'very good', 2,344 (47.3\%) as 'good', 1,473 (29.7\%) as 'fair', $483(9.7 \%)$ as 'poor', and $151(3.0 \%)$ as 'very poor'. Participants in the lower categories of SRH tended to be older, female, widowed, less educated, scored lower in the MMSE, and had higher functional dependence (as assessed by the Pfeffer FAQ scale). Self-reported health status was also associated to the geographical 
Table 1. Baseline demographic and clinical characteristics by categories of global SRH in the NEDICES Study cohort $(\mathrm{n}=4,958)$

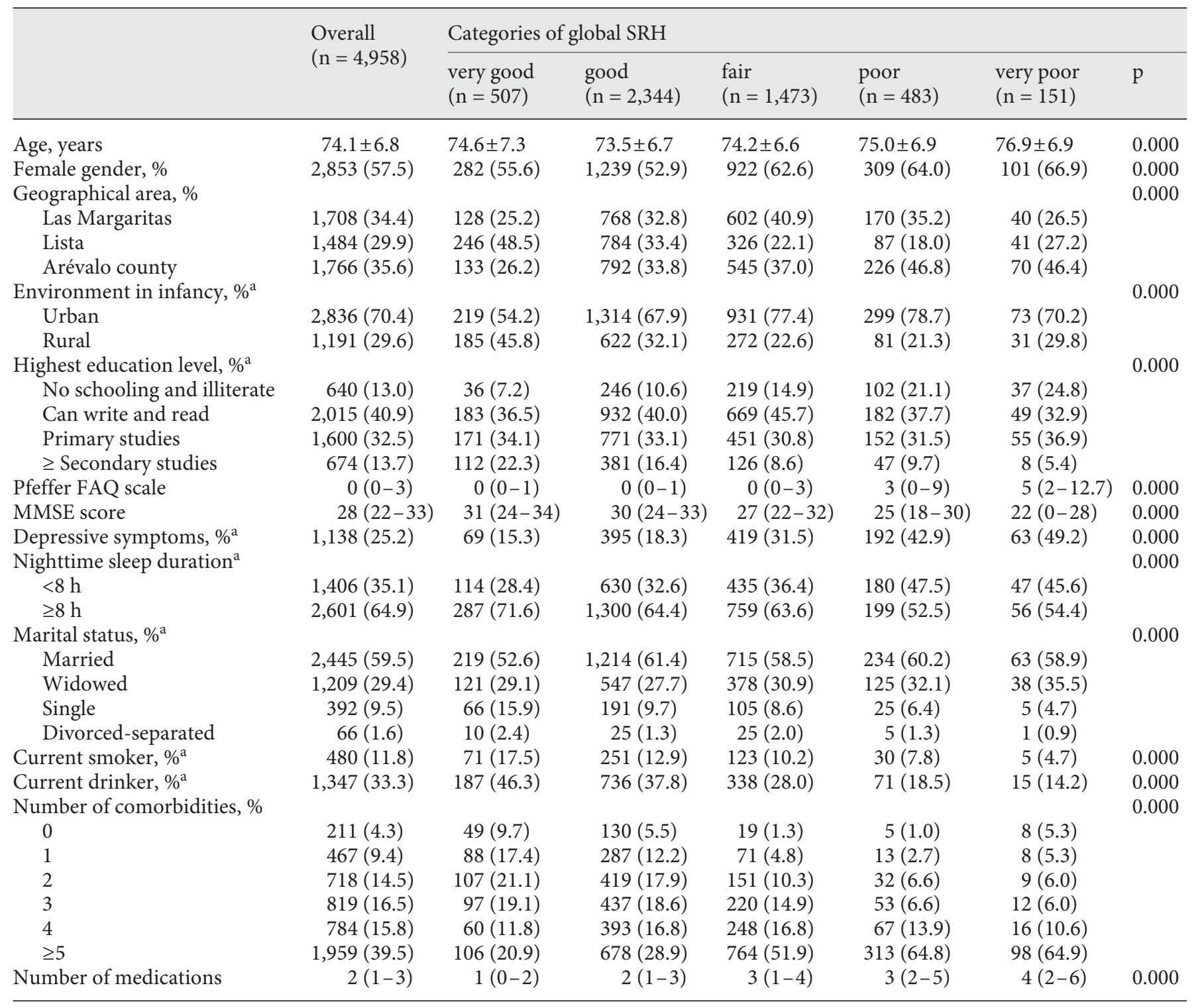

Mean value \pm SD is given for age; median values (25th-75th percentile) are given for Pfeffer FAQ score, MMSE score, and number of medications. ${ }^{\mathrm{a}} \mathrm{n}<4,958$ because of missing data.

area, with better health perception among participants living in Lista (white-collar area), and worse among those living in Arévalo (rural area). Subjects with a higher number of chronic comorbidities and medications were more likely to report poorer SRH.

Follow-up time for the entire cohort was 48,946 person-years. Seven participants (0.1\%) lacked data on vital status and were excluded from mortality analysis. At the end of observation, 2,468 of 4,951 participants (49.8\%) had died, with an overall mean survival of 10.03 years (95\% CI: 9.91-10.15). As shown in table 2, the most commonly reported causes of mortality were cancer $(24.7 \%$ of deaths), other cardiovascular diseases (17.9\%), and respiratory diseases (14.5\%).

The association between SRH and all-cause mortality is shown in table 3. Lower categories in both global and age-comparative SRH were independently associated with a worse survival, even after adjusting for sociodemo- 
Table 2. Specific causes of death among participants in the NEDICES Study cohort

\begin{tabular}{lll}
\hline Specific cause of death & Number & $\begin{array}{l}\text { Proportion of mortality } \\
(\%)^{\mathrm{a}}\end{array}$ \\
\hline Cancer & 609 & 24.7 \\
Other cardiovascular diseases & 443 & 17.9 \\
Respiratory diseases & 359 & 14.5 \\
Myocardial infarction & 280 & 11.3 \\
Stroke & 203 & 8.2 \\
Dementia & 133 & 5.4 \\
Other neurological disorders & 36 & 1.5 \\
Other causes & 405 & 16.4 \\
\hline
\end{tabular}

${ }^{a}$ Percentage based on number of deceased participants at the end of the follow-up pe$\operatorname{riod}(\mathrm{n}=2,468)$.

Table 3. Association between SRH and all-cause mortality between 1994 and 2007

\begin{tabular}{|c|c|c|c|c|c|c|c|}
\hline & Deaths $(\%)^{\mathrm{a}}$ & \multicolumn{2}{|c|}{ Model $1^{b}$} & \multicolumn{2}{|c|}{ Model $2^{c}$} & \multicolumn{2}{|c|}{ Model $3^{\mathrm{d}}$} \\
\hline Very good & $237(46.7)$ & 1 & - & 1 & - & 1 & - \\
\hline Good & $1,008(43.0)$ & 0.98 & $0.85-1.13$ & 0.97 & $0.83-1.14$ & 0.95 & $0.81-1.12$ \\
\hline Fair & $797(54.2)$ & $1.45^{\ddagger}$ & $1.25-1.68$ & $1.32^{\dagger}$ & $1.12-1.55$ & $1.22^{*}$ & $1.03-1.44$ \\
\hline Much better & $158(47.7)$ & 1 & - & 1 & - & 1 & - \\
\hline Better & $704(45.5)$ & 1.07 & $0.90-1.28$ & 1.06 & $0.88-1.26$ & 1.05 & $0.88-1.25$ \\
\hline Similar & $776(47.9)$ & $1.32^{\dagger}$ & $1.11-1.57$ & $1.27^{\dagger}$ & $1.06-1.52$ & $1.22^{*}$ & $1.02-1.46$ \\
\hline Worse/much worse & $331(63.4)$ & $2.28^{\ddagger}$ & $1.89-2.77$ & $1.90^{\ddagger}$ & $1.55-2.33$ & $1.73^{\ddagger}$ & $1.41-2.12$ \\
\hline \multicolumn{8}{|l|}{ Time-comparative SRH ${ }^{\mathrm{f}}$} \\
\hline
\end{tabular}

${ }^{a}$ Percentages based on number of participants for whom vital status was available $(\mathrm{n}=4,951) .{ }^{\mathrm{b}}$ Model 1 adjusted for gender and age. ${ }^{\mathrm{c}}$ Model 2 adjusted for gender, age, education level, marital status, Pfeffer FAQ scale, MMSE score, and current smoking and alcohol consumption. ${ }^{\mathrm{d}}$ Model 3 adjusted for gender, age, education level, marital status, Pfeffer FAQ scale, MMSE score, current smoking, alcohol consumption, and number of chronic comorbidities. ${ }^{\mathrm{e}}$ Data of age-comparative SRH available for 4,023 participants. ${ }^{\mathrm{f}}$ Data of time-comparative SRH available for 4,038 participants. ${ }^{*}$ Significant at $\mathrm{p}<0.05 ;{ }^{\dagger}$ significant at $\mathrm{p}<0.01 ;{ }^{\ddagger}$ significant at $\mathrm{p}<0.001$.

graphic variables, functional status and number of comorbid conditions. In detail, those participants with 'poor or very poor' global SRH had a more than 2 -fold greater hazard of death compared with those who perceived their health as 'very good' (reference category) in the age- and gender-adjusted model. Although the inclusion of other covariates into the Cox model reduced such an effect, a significant risk for all-cause mortality remained among participants with 'fair' (aHR 1.22; 95\% CI: 1.03-1.44) and 'poor or very poor' global SRH (aHR 1.39; 95\% CI: 1.151.69). In terms of comparative SRH measurements, subjects considering their health as 'worse or much worse' than those of the same age and gender (age-comparative SRH) had an aHR for all-cause mortality of 1.73 (95\% CI: 1.41-2.12). Finally, participants who rated their current health as 'similar' to that in the previous year (time-comparative SRH) exhibited a lower risk of dying (aHR 0.83; 95\% CI: 0.72-0.94) compared to the reference category. 
Table 4. Adjusted hazard ratios (with 95\% CIs) for cause-specific mortality according to different measurements of SRH

\begin{tabular}{|c|c|c|c|c|c|c|}
\hline & \multicolumn{6}{|c|}{ Specific causes of death } \\
\hline & cancer & cardiovascular diseases & respiratory diseases & stroke & dementia & other causes \\
\hline \multicolumn{7}{|l|}{ Global SRH } \\
\hline Very good & 1 & 1 & 1 & 1 & 1 & 1 \\
\hline Good & $0.84(0.62-1.12)$ & $1.11(0.81-1.52)$ & $1.07(0.68-1.67)$ & $1.41(0.78-2.56)$ & $0.70(0.39-1.26)$ & $0.82(0.56-1.19)$ \\
\hline Fair & $1.07(0.78-1.48)$ & $1.36(0.98-1.89)$ & $1.93(1.22-3.06)^{\dagger}$ & $1.13(0.59-2.13)$ & $0.93(0.50-1.74)$ & $1.02(0.68-1.52)$ \\
\hline Poor/very poor & $0.93(0.61-1.42)$ & $1.45(0.99-2.11)$ & $2.61(1.55-4.39)^{\ddagger}$ & $1.49(0.74-3.01)$ & $1.41(0.70-2.84)$ & $1.32(0.83-2.09)$ \\
\hline \multicolumn{7}{|l|}{ Age-comparative SRH } \\
\hline Much better & 1 & 1 & 1 & 1 & 1 & 1 \\
\hline Better & $1.00(0.71-1.41)$ & $0.89(0.65-1.22)$ & $0.93(0.59-1.49)$ & $2.40(1.03-5.58)^{*}$ & $0.99(0.47-2.06)$ & $1.25(0.79-1.98)$ \\
\hline Similar & $1.09(0.77-1.54)$ & $1.09(0.79-1.49)$ & $1.35(0.86-2.13)$ & $2.60(1.11-6.10)^{*}$ & $1.51(0.73-3.13)$ & $1.18(0.74-1.89)$ \\
\hline Worse/much worse & $1.11(0.71-1.72)$ & $1.52(1.06-2.18)^{*}$ & $2.45(1.47-4.07)^{\dagger}$ & $3.66(1.49-9.03)^{\dagger}$ & $1.54(0.66-3.58)$ & $2.21(1.32-3.70)^{*}$ \\
\hline \multicolumn{7}{|l|}{ Time-comparative SRH } \\
\hline Much better/better & 1 & 1 & 1 & 1 & 1 & 1 \\
\hline Similar & $0.68(0.53-0.88)^{*}$ & $0.83(0.64-1.07)$ & $0.83(0.59-1.17)$ & $0.95(0.60-1.50)$ & $0.68(0.40-1.16)$ & $1.09(0.77-1.56)$ \\
\hline Worse/much worse & $0.79(0.59-1.05)$ & $1.16(0.89-1.52)$ & $1.00(0.69-1.45)$ & $0.89(0.55-1.47)$ & $1.22(0.71-2.09)$ & $1.05(0.72-1.53)$ \\
\hline
\end{tabular}

All models are adjusted for gender, age, education level, marital status, Pfeffer FAQ scale, MMSE score, current smoking, alcohol consumption, and number of chronic comorbidities. * Significant at $\mathrm{p}<0.05 ;{ }^{\dagger}$ significant at $\mathrm{p}<0.01{ }^{\ddagger}{ }^{\ddagger}$ significant at $\mathrm{p}<0.001$.

The association between SRH and cause-specific mortality is shown in table 4 . In short, global SRH was an independent predictor only for death due to respiratory diseases - and showed a near significant association with death due to cardiovascular diseases - whereas age-comparative SRH exhibited a strong gradient effect on the risk of death due to stroke. Time-comparative SRH had an independent effect only on death from cancer. Due to low relative frequencies, 'myocardial infarction' and 'other cardiovascular diseases' were merged into one cause of death ('cardiovascular diseases') for the analysis, and 'other neurological disorders' was included into the category of 'dementia'. In depth, global SRH exerted an independent effect on the mortality due to respiratory diseases, with aHRs of 1.93 and 2.61 for participants with 'fair' and 'poor or very poor' SRH, respectively, compared to those with 'very good' status (reference category). We also found a near significant association between global SRH and mortality due to cardiovascular causes (aHR 1.45; 95\% CI: $0.99-2.11$ for participants in the 'poor or very poor' category). On the other hand, age-comparative SRH was a strong predictor of mortality due to stroke with a clear gradient in aHRs across almost all response categories, from 2.40 for 'better' to 3.66 for 'worse or much worse' categories - and to a lesser extent of mortality due to cardiovascular diseases, respiratory diseases, and other causes - with significant associations only for the lowest health category. With regard to the time-comparative question, participants who rated their current

Self-Rated Health and Cause-Specific Mortality status as 'similar' in comparison to the previous year had a lower mortality due to cancer (aHR 0.68; 95\% CI: $0.53-$ 0.88 ) compared to the reference category ('much better or better').

\section{Discussion}

In this population-based survey of community-dwelling elderly individuals we found that the prognostic value for mortality of subjective health largely varies according to the cause of death and the type of measurement evaluated. Global SRH exerted an independent association only with the risk of death from respiratory diseases and, nearing statistical significance, from cardiovascular diseases - whereas age-comparative SRH revealed itself as a strong predictor for death from stroke and - with a weaker association - from respiratory and cardiovascular diseases and other causes.

Few authors had previously addressed the predictive power of SRH for cause-specific mortality, with somewhat contradictory results. Tsuji et al. [17] found a significant association between SRH and cancer mortality in a cohort of Japanese elderly individuals. Although SRH was also found to be a predictor for stroke mortality after adjusting for age and gender, this variable lost statistical significance when functional disability was entered into the multivariate model [17]. Similar findings were reported from a Dutch study in which SRH exhibited an independent pre- 
dictive effect only for death due to non-cardiovascular causes [16]. Giltay et al. [22] have recently confirmed these results in a subanalysis of this cohort with a longer followup period. On the opposite, other studies [4, 18-20] have demonstrated a clear association between subjective health and cardiovascular mortality, even after adjusting for diverse cardiovascular risk factors and biological variables (e.g., cholesterol level or systolic blood pressure). However, it should be noted that most of these cohort studies only took into account a low number of different causes of death - namely malignant and cardiovascular diseases [16-20]. To our knowledge, only one previous study is comparable to ours in terms of detailed description of the cause-specific mortality [21]. Benjamins et al. [21] analyzed mortality data from the National Death Index in a cohort of over 600,000 noninstitutionalized U.S. adults. The underlying causes of mortality were similar to those in the NEDICES Study cohort, with heart disease (35.6\%) and cancer (26.7\%) accounting for the majority of deaths. SRH most strongly predicted mortality due to diabetes, respiratory diseases, and infectious causes (with aHRs ranging from 6.12 to 3.68 for those reporting poor $\mathrm{SRH}$ compared to those with excellent SRH), whereas a more moderate association was demonstrated with deaths due to heart disease, cancer, and stroke. Consistently, the authors found very weak or nonexistent relationships with mortality secondary to 'external causes' unconnected with physical status (e.g., accidents or suicide) [21]. As a result of significant gender interaction effects, being male was associated with an increased risk of mortality for certain causes across the diverse categories of SRH.

In the present study, contrary to that by Benjamins et al. [21], the only cause of death independently predicted by global SRH was respiratory diseases. Although the specific diagnosis of the underlying condition could not be ascertained from death certificate data, it seems likely that COPD accounted for most of them. Health status, as assessed by health-related quality of life (HRQL) questionnaires, correlates with respiratory mortality, but not with mortality of other causes, in patients diagnosed with COPD $[30,31]$. In addition, the frequency of exacerbations - a marker of COPD severity - is linked with meaningful decreases in HRQL scores [32]. As previously suggested [21], it could be hypothesized that duration of illness plays a capital role in the magnitude of relationship between SRH and mortality. The slowly progressive natural history of COPD is well documented [33]. Even patients with undiagnosed COPD in early stages already show impairment in HRQL [34], whereas data from large COPD trials suggest that nonmalignant respiratory dis- ease is the predominant cause of death only in patients with mild to moderate stages [35]. Interestingly, we also found a strong trend - although not statistically significant - towards an association between the lower categories of SRH and mortality from cardiovascular causes. Such a finding is in line with previous studies $[4,8,18-20]$ and reinforces the idea that the longer the course of the condition that ultimately leads to death and the greater its impact on HRQL (e.g., heart failure), the stronger the association between SHR and cause-specific mortality appears to be. In the same vein, Idler et al. [36] found that the association between global SRH and all-cause mortality was stronger among those respondents who had experiential knowledge of chronic circulatory system illness as compared to healthy individuals, thus reinforcing the idea that self-ratings of health are based on and reinforced by own first-hand experiences with illness. In contrast, malignant diseases usually exhibit a prolonged subclinical phase and its impact on HRQL and daily activities tend to be concentrated in more advanced stages, which directly contribute to the subject's death. It is possible that the differences observed between the present study and that by Benjamins et al. [21] in the predictive power of global SRH can be partially explained by the lack of adjusting for the effect of the number of chronic comorbidities - that is, the subject's objective health status - in the latter. This fact might have contributed to overestimate the independent impact of this variable on some specific causes of death. Participants with higher number of selfreported chronic conditions were more likely to perceive their health as poorer both in the present cohort and in others $[9,22]$. In addition, the strength of the association between the different measurements of SRH and all-cause mortality diminished when the number of comorbidities was entered into the Cox model.

As another main result of the present paper, we found that different subjective health measurements had a variable impact on cause-specific mortality. Age-comparative SRH exhibited a higher predictive accuracy than global SRH for death due to cardiovascular diseases, other causes, and particularly stroke - for which the variable showed a 'dose-dependent' effect. Indeed, even participants reporting a 'better' age-comparative SRH were more likely to die from stroke as compared to those in the reference category ('much better'), with an aHR of 2.40 , whereas the risk for participants with 'worse or much worse' health was more than 3.5-fold higher. It has been reported that poorer self-rated physical and mental health status increased the risk of vascular mortality in patients with symptomatic and asymptomatic atherosclerotic dis- 
ease [8]. Only a small number of studies have compared the relationship between the different formulations of SRH and mortality [19, 37-40]. The overall agreement between the questions 'How would you describe your general health lately?' and 'Compared to men/women your age how would you define your health?' has been demonstrated to be far from excellent [41]. Nevertheless, the prediction of survival by age-comparative SRH did not differ significantly from global SRH in elderly men [36]. In line with this and other studies [38], we found that both measurements were equally predictors of all-cause mortality with similar aHRs between analogous answer categories. With regard to cause-specific mortality, Appels et al. [19] also reported that the age-comparative question was associated much more strongly with mortality from cardiovascular diseases and coronary heart disease compared with the global question. Similarly, cardiovascular mortality was independently predicted by age-comparative but not global SRH in a populationbased cohort from Germany [4]. Comparative SRH measurements demand from the respondent to create a reference frame by estimating the health of a group of agecomparable subjects, and may be influenced by the perception that the individual has of the community that he/she belongs to. As recently pointed out, the age sensitivity of these evaluations imposes caution in predicting mortality in elderly populations [39]. Moreover, differences in the exact wording of the questions and the number of answer categories make direct comparisons between studies focused on comparative SRH difficult.

In overall terms, time-comparative $\mathrm{SRH}$ provided small additional predictive value in our cohort as compared to global or age-comparative items, similarly to previous studies [39, 40]. Sargent-Cox et al. [39] reported that the self-comparative evaluation of SRH ('Is your health now better, about the same or not as good as it was 12 months ago?') was the weakest predictor of mortality among three different measurements. As a somewhat counterintuitive result we found that participants who rated their health status as 'similar' in comparison to the previous year had lower aHRs for all-cause and cancerrelated mortality than those in the 'much better or better' category. Other authors also reported a similar association for all-cause mortality [39]. It could be hypothesized that those subjects who stated that their status was better were actually reporting an - at least partial - recovery from a recent health issue that might subsequently increase mortality risk. Otherwise, participants in the 'same' time-comparative SRH group would reflect stability in their health status over time.

Self-Rated Health and Cause-Specific Mortality
The fact that neither global nor age-comparative SRH evaluations were found to be associated to the risk of death from cancer or dementia in our study is noteworthy. As suggested above, the symptomatic phase of those malignancies aggressive enough to lead to death uses to be short and late-onset. On the other hand, patients with Alzheimer's disease and other neurodegenerative dementias are frequently unaware of their cognitive symptoms and medical diagnosis [42]. Therefore, it is intuitive that the relative impact of SRH in both settings may be moderated by other stronger biological or psychosocial variables. Furthermore, the potentially deleterious effect of poor self-perceived health status on lifestyle behaviors (e.g., decrease in leisure-time physical activity) [43] seems to be more decisive in the prognosis of cardiovascular or respiratory diseases.

Our paper has some limitations. Although SRH was measured twice during the follow-up period, we only analyzed the impact of the baseline evaluation. The dynamic assessment of subjective health trajectories over time has been revealed as an even stronger predictor of mortality than baseline assessment [44]. Despite the very large sample size, some associations between SRH and specific causes of death were probably nonsignificant due to insufficient statistical power. It should also be noted that the reference group in the cause-specific mortality analysis (table 4) included both survivors and those participants who died from other causes, thus contributing to dilute to some extent the strength of such associations. We are not able to exclude the existence of inaccuracies in selfreport secondary to misunderstanding by participants regarding their previous diagnosis or the manner in which the questionnaire was applied. Finally, the lack of accuracy of the information provided by the death certificates may potentially lead to bias in reporting cause-specific mortality.

This study also exhibits considerable strengths because of its large size, high response rate for the SRH questions, prospective design, and sizable set of variables considered for adjustment. Unlike previous cohorts [4, 16-20], we analyzed a comprehensive set of specific causes of death. The NEDICES Study was conducted in three well-defined community-based areas and the participation rate at baseline was notably high. Although we are unable to provide any census comparison with the general Spanish population, educational attainment, occupation and health status data in the NEDICES Study are analogous to other Spanish studies performed in the elderly [45]. Moreover, the reliability and consistency of its data collection procedures have been well established 
$[23-25,29]$. Finally, we had complete death information of almost the entire cohort (99.8\%).

In conclusion, we have found that the ability of SRH in predicting mortality among community-dwelling elderly individuals largely differs according to the specific cause of death and the way it is elicited. Global SRH at baseline was a significant predictor for mortality due to respiratory and cardiovascular diseases, whereas agecomparative SRH emerged as a strong predictor of mortality due to stroke with a clear gradient across different answer categories. As a whole, our findings suggest that the predictive value of the different measurements of SRH is more evident if the condition that ultimately leads to death exhibits a protracted clinical course and a notable impact on daily life and HRQL. On the opposite, the predictive accuracy of these variables is suboptimal in those more aggressive conditions with poor prognosis in the mid-term or if the responder has insufficient self-awareness of own functional performance decrements. Moreover, the time-comparative question provides only little additional predictive power to the global or age-comparative measurements. Further investigations should take into account these different explanatory mechanisms by which subjective health predicts survival in order to clarify their underlying determinants.

\section{Grants and Financial Support}

This work was partly supported by the Fundación Mutua Madrileña (2006-2009); the Spanish Health Research Agency (FIS 93/0773); the Centro de Investigación Biomédica en Red sobre Enfermedades Neurodegenerativas (CIBERNED); and the National Institutes of Health (NIH R01 NS039422 to J.B.-L. and F.B.-P.). M.F.-R. holds a research training contract 'Rio Hortega' (CM11/00187) from the Spanish Ministry of Economy and Competitiveness (Instituto de Salud Carlos III).

\section{Acknowledgements}

A complete list of the investigators and advisors of the NEDICES Study group is exposed in www.ciberned.es and www. neurologia12o.com.

\section{Disclosure Statement} close.

\section{References}

1 Mossey JM, Shapiro E: Self-rated health: a predictor of mortality among the elderly. Am J Public Health 1982;72:800-808.

2 Idler EL, Benyamini Y: Self-rated health and mortality: a review of twenty-seven community studies. J Health Soc Behav 1997;38:21-37.

3 McGee DL, Liao Y, Cao G, Cooper RS: Selfreported health status and mortality in a multiethnic US cohort. Am J Epidemiol 1999;149: 41-46.

4 Heidrich J, Liese AD, Löwel H, Keil U: Selfrated health and its relation to all-cause and cardiovascular mortality in southern Germany. Results from the MONICA Augsburg cohort study 1984-1995. Ann Epidemiol 2002; 12:338-345.

5 Singh-Manoux A, Dugravot A, Shipley MJ, Ferrie JE, Martikainen P, Goldberg M, Zins M; GAZEL Cohort: The association between self-rated health and mortality in different socioeconomic groups in the GAZEL cohort study. Int J Epidemiol 2007;36:1222-1228.

6 Nielsen AB, Siersma V, Hiort LC, Drivsholm T, Kreiner S, Hollnagel H: Self-rated general health among 40-year-old Danes and its association with all-cause mortality at 10-,20-, and 29-years' follow-up. Scand J Public Health 2008;36:3-11.
7 DeSalvo KB, Bloser N, Reynolds K, He J, Muntner P: Mortality prediction with a single general self-rated health question. A metaanalysis. J Gen Intern Med 2006;21:267-275.

-8 Grool AM, van der Graaf Y, Visseren FL, de Borst GJ, Algra A, Geerlings MI; on behalf of the SMART Study Group: Self-rated health status as a risk factor for future vascular events and mortality in patients with symptomatic and asymptomatic atherosclerotic disease: the SMART study. J Intern Med 2012;272:277-286.

-9 Thong MS, Kaptein AA, Benyamini Y, Krediet RT, Boeschoten EW, Dekker FW; Netherlands Cooperative Study on the Adequacy of Dialysis (NECOSAD) Study Group: Association between a self-rated health question and mortality in young and old dialysis patients: a cohort study. Am J Kidney Dis 2008;52:111-117.

10 Idler EL, Kasl SV: Self-ratings of health: do they also predict change in functional ability? J Gerontol B Psychol Sci Soc Sci 1995;50:S344S353.

11 Fernández-Olano C, Hidalgo JD, Cerdá-Díaz R, Requena-Gallego M, Sánchez-Castaño C, Urbistondo-Cascales L, Otero-Puime A: Factors associated with health care utilization by the elderly in a public health care system. Health Policy 2006;75:131-139.
2 Fayers PM, Sprangers MA: Understanding self-rated health. Lancet 2002;359:187-188.

13 Helweg-Larsen M, Kjøller M, Thoning H: Do age and social relations moderate the relationship between self-rated health and mortality among adult Danes? Soc Sci Med 2003;57: 1237-1247.

14 Okamoto K, Momose Y, Fujino A, Osawa Y: Gender differences in the relationship between self-rated health (SRH) and 6-year mortality risks among the elderly in Japan. Arch Gerontol Geriatr 2008;47:311-317.

15 Regidor E, Guallar-Castillón P, Gutiérrez-Fisac JL, Banegas JR, Rodríguez-Artalejo F: Socioeconomic variation in the magnitude of the association between self-rated health and mortality. Ann Epidemiol 2010;20:395-400.

16 Pijls LT, Feskens EJ, Kromhout D: Self-rated health, mortality, and chronic diseases in elderly men. The Zutphen Study, 1985-1990. Am J Epidemiol 1993;138:840-848.

-17 Tsuji I, Minami Y, Keyl PM, Hisamichi S, Asano H, Sato M, Shinoda K: The predictive power of self-rated health, activities of daily living, and ambulatory activity for cause-specific mortality among the elderly: a three-year follow-up in urban Japan. J Am Geriatr Soc 1994;42:153-156. 
-18 Kaplan GA, Goldberg DE, Everson SA, Cohen RD, Salonen R, Tuomilehto J, Salonen J: Perceived health status and morbidity and mortality: evidence from the Kuopio ischaemic heart disease risk factor study. Int J Epidemiol 1996;25:259-256.

-19 Appels A, Bosma H, Grabauskas V, Gostautas A, Sturmans F: Self-rated health and mortality in a Lithuanian and a Dutch population. Soc Sci Med 1996;42:681-689.

20 Heistaro S, Jousilahti P, Lahelma E, Vartiainen E, Puska P: Self rated health and mortality: a long term prospective study in eastern Finland. J Epidemiol Community Health 2001; 55:227-232.

-21 Benjamins MR, Hummer RA, Eberstein IW, Nam CB: Self-reported health and adult mortality risk: an analysis of cause-specific mortality. Soc Sci Med 2004;59:1297-1306.

22 Giltay EJ, Vollaard AM, Kromhout D: Selfrated health and physician-rated health as independent predictors of mortality in elderly men. Age Ageing 2012;41:165-171.

-23 Benito-Leon J, Bermejo-Pareja F, Morales JM, Vega S, Molina JA: Prevalence of essential tremor in three elderly populations of central Spain. Mov Disord 2003;18:389-394.

-24 Díaz-Guzmán J, Bermejo-Pareja F, BenitoLeón J, Vega S, Gabriel R, Medrano MJ; Neurological Disorders in Central Spain (NEDICES) Study Group: Prevalence of stroke and transient ischemic attack in three elderly populations of central Spain. Neuroepidemiology 2008;30:247-253.

25 Posada IJ, Benito-León J, Louis ED, Trincado R, Villarejo A, Medrano MJ, Bermejo-Pareja F: Mortality from Parkinson's disease: a population-based prospective study (NEDICES). Mov Disord 2011;26:2522-2529.

26 Encuesta Nacional de Salud (ENS) de España. Ministerio de Sanidad, Servicios Sociales e Igualdad. Madrid, 2012. Available at: http:// www.msps.es/estadEstudios/estadisticas/ encuestaNacional/encuesta1997.htm. cessed on July 8, 2012.
27 Folstein MF, Folstein SE, McHugh PR: 'Minimental state'. A practical method for grading the cognitive state of patients for the clinician. J Psychiatr Res 1975;12:189-198.

28 Pfeffer RI, Kurosaki TT, Harrah CH Jr, Chance JM, Filos S: Measurement of functional activities in older adults in the community. J Gerontol 1982;37:323-329.

29 Louis ED, Benito-León J, Bermejo-Pareja F; Neurological Disorders in Central Spain (NEDICES) Study Group: Self-reported depression and anti-depressant medication use in essential tremor: cross-sectional and prospective analyses in a population-based study. Eur J Neurol 2007;14:1138-1146.

30 Marin JM, Cote CG, Diaz O, Lisboa C, Casanova C, Lopez MV, Carrizo SJ, Pinto-Plata V, Dordelly LJ, Nekach H, Celli BR: Prognostic assessment in COPD: health related quality of life and the BODE index. Respir Med 2011; 105:916-921.

31 Frostad A, Soyseth V, Haldorsen T, Andersen A, Gulsvik A: Respiratory symptoms and 30 year mortality from obstructive lung disease and pneumonia. Thorax 2006;61:951-956.

32 Anzueto A, Leimer I, Kesten S: Impact of frequency of COPD exacerbations on pulmonary function, health status and clinical outcomes. Int J Chron Obstruct Pulmon Dis 2009;4:245-251.

33 Fletcher C, Peto R, Tinker C, Speizer F: The Natural History of Chronic Bronchitis and Emphysema. New York, Oxford University Press, 1976.

34 Miravitlles M, Soriano JB, García-Río F, Muñoz L, Duran-Tauleria E, Sanchez G, Sobradillo V, Ancochea J: Prevalence of COPD in Spain: impact of undiagnosed COPD on quality of life and daily life activities. Thorax 2009; 64:863-868.

35 Berry CE, Wise RA: Mortality in COPD: causes, risk factors, and prevention. COPD 2010;7:375-382.

36 Idler E, Leventhal H, McLaughlin J, Leventhal E: In sickness but not in health: self-ratings, identity, and mortality. J Health Soc Behav 2004;45:336-356.
37 Baron-Epel O, Shemy G, Carmel S: Prediction of survival: a comparison between two subjective health measures in an elderly population. Soc Sci Med 2004;58:2035-2043.

38 Vuorisalmi M, Lintonen T, Jylhä M: Global self-rated health data from a longitudinal study predicted mortality better than comparative self-rated health in old age. J Clin Epidemiol 2005;58:680-687.

39 Sargent-Cox KA, Anstey KJ, Luszcz MA: The choice of self-rated health measures matter when predicting mortality: evidence from 10 years follow-up of the Australian longitudinal study of ageing. BMC Geriatr 2010;10:18.

40 Bath PA: Differences between older men and women in the self-rated health-mortality relationship. Gerontologist 2003;43:387-395; discussion 372-375.

41 Baron-Epel O, Kaplan G: General subjective health status or age-related subjective health status: does it make a difference? Soc Sci Med 2001;53:1373-1381.

42 Zamboni G, Wilcock G: Lack of awareness of symptoms in people with dementia: the structural and functional basis. Int J Geriatr Psychiatry 2011;26:783-792.

43 Zimmermann E, Ekholm O, Grønbaek M, Curtis T: Predictors of changes in physical activity in a prospective cohort study of the Danish adult population. Scand J Public Health 2008;36:235-241.

44 Ferraro KF, Kelley-Moore JA: Self-rated health and mortality among black and white adults: examining the dynamic evaluation thesis. J Gerontol B Psychol Sci Soc Sci 2001; 56:S195-S205.

45 Morales JM, Bermejo FP, Benito-León J, Rivera-Navarro J, Trincado R, Gabriel SR, Vega S; NEDICES Study Group: Methods and demographic findings of the baseline survey of the NEDICES cohort: a door-to-door survey of neurological disorders in three communities from Central Spain. Public Health 2004; 118:426-433.
Self-Rated Health and Cause-Specific Mortality
Gerontology 2013;59:368-377 DOI: $10.1159 / 000348781$ 